Document downloaded from:

http://hdl.handle.net/10251/161607

This paper must be cited as:

Chicharro Lopez, FI.; Cordero Barbero, A.; Garrido, N.; Torregrosa Sánchez, JR. (2020). On the choice of the best members of the Kim family and the improvement of its convergence. Mathematical Methods in the Applied Sciences. 43(14):8051-8066.

https://doi.org/10.1002/mma.6014

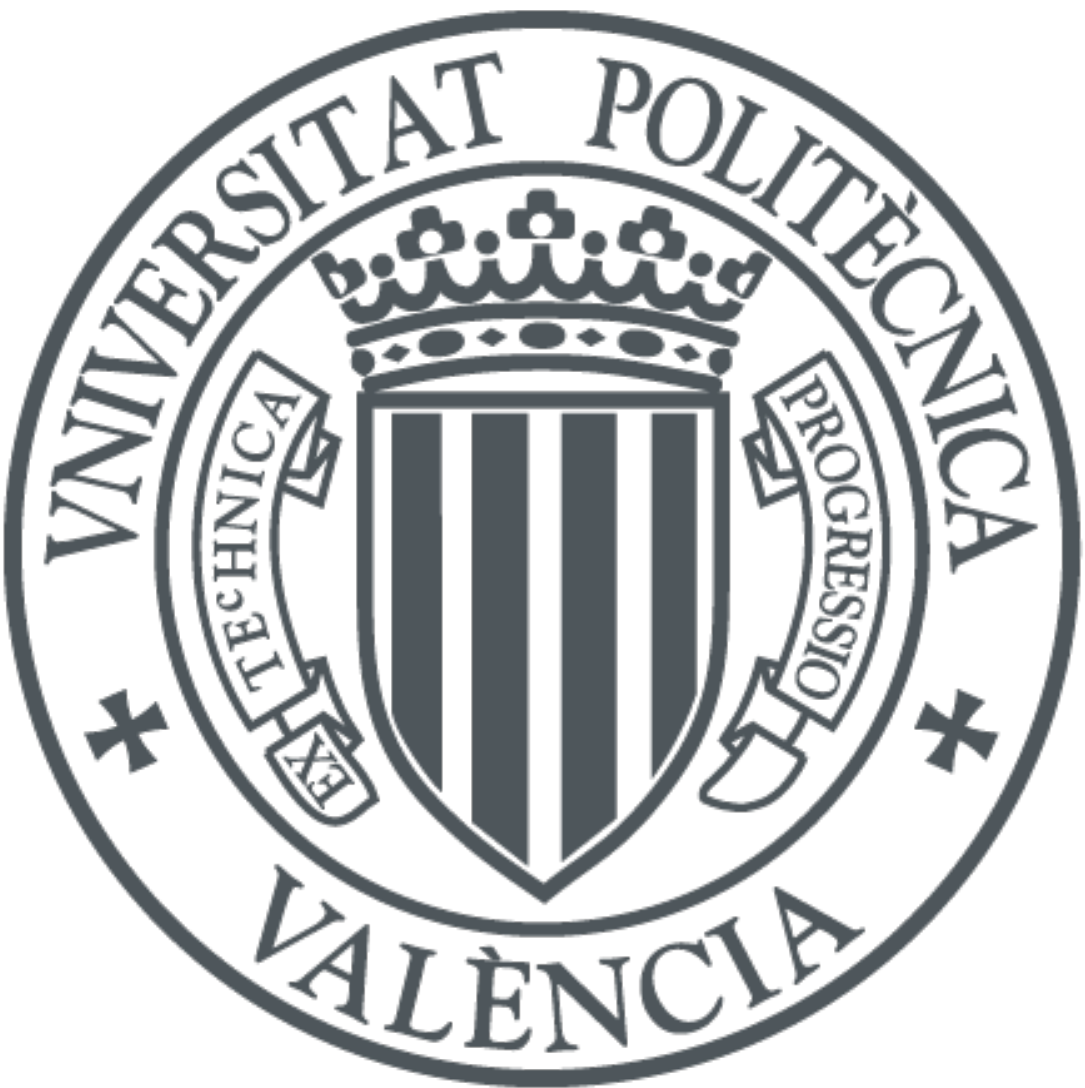

The final publication is available at

https://doi.org/10.1002/mma.6014

Copyright John Wiley \& Sons

Additional Information 


\title{
On the choice of the best members of the Kim family and the improvement of its convergence
}

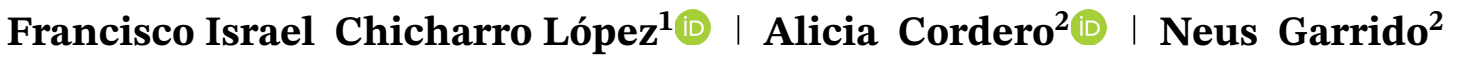 \\ Juan Ramon Torregrosa ${ }^{2}$
}

\author{
${ }^{1}$ Escuela Superior de Ingeniería y \\ Tecnología, Universidad Internacional de \\ La Rioja, Logroño, Spain \\ ${ }^{2}$ Institute for Multidisciplinary \\ Mathematics, Universitat Politècnica de \\ València, València, Spain

\section{Correspondence} \\ Alicia Cordero, Institute for \\ Multidisciplinary Mathematics, \\ Universitat Politècnica de Valéncia, \\ Camino de Vera s/n, Valéncia 46022, \\ Spain. \\ Email: acordero@mat.upv.es \\ Communicated by: W. Sprößig \\ Funding information \\ Generalitat Valenciana, Grant/Award \\ Number: PROMETEO/2016/089; \\ Ministerio de Ciencia e Innovación, \\ Grant/Award Number: \\ PGC2018-095896-B-C22
}

The best members of the Kim family, in terms of stability, are obtained by using complex dynamics. From this elements, parametric iterative methods with memory are designed. A dynamical analysis of the methods with memory is presented in order to obtain information about the stability of them. Numerical experiments are shown for confirming the theoretical results.

\section{KEYWORDS}

basin of attraction, iterative methods with memory, low-dimensional dynamical systems, nonlinear algebraic or transcendental equations, parameter plane, stability

\section{MSC CLASSIFICATION}

$65 \mathrm{H} 05$

\section{1 | INTRODUCTION}

To find a solution $\xi$ of the nonlinear equation $f(x)=0$ is a common problem that appears frequently in different fields of science and engineering. These nonlinear problems require iterative methods to be solved. In the last decades, there has been an extensive literature focused on the generation of iterative procedures to find a solution $\xi$. We can see a good overview on classical and recent results in Amat and Busquier ${ }^{1}$ or Petković et al. ${ }^{2}$

The iterative methods are fixed-point schemes that, starting from one or more initial estimations, obtain a new value that approaches our solution as

$$
x_{k+1}=g\left(x_{k-p}, x_{k-p+1}, \ldots, x_{k-1}, x_{k}\right), \quad k \geq 1, \quad p \geq 0 .
$$

If we use only the last iteration ( $p=0$ ), we have an iterative method without memory and, if we use more than one previous iterations $(p>0)$, the iterative scheme is with memory.

Related with the order of convergence of iterative methods without memory, the Kung-Traub conjecture ${ }^{3}$ establishes that the order of a scheme without memory is always lower than $2^{d-1}$, where $d$ is the number of functional evaluations per iteration. When this bound is reached, the iterative method is called optimal. One technique for improving the order of convergence without increasing the number of functional evaluations is to introduce memory in the scheme, that is, the new iterate depend on more than one previous iterates. 
The first method with memory including accelerators parameters is based on Steffensen's method ${ }^{4}$ and was designed by Traub. ${ }^{5} \mathrm{He}$ constructed a method with memory with slight but interesting changes of Steffensen's scheme, resulting in a method with order of convergence 2.41 .

There is an extensive literature of methods with memory that include derivatives in their iterative expressions (see, for example, Petković et $\mathrm{al}^{2}$ and the references therein) and other ones that are derivative free, such as the papers of Petković and Dz̃unić6-8 or by other authors, ${ }^{9-13}$ all of them by using similar techniques.

In Kim, ${ }^{14}$ a three-step family of eighth-order iterative methods depending on three parameters is introduced. If we only focus on the two-step family, the iterative scheme is

$$
\begin{aligned}
y_{k} & =x_{k}-\frac{f\left(x_{k}\right)}{f^{\prime}\left(x_{k}\right)}, \\
x_{k+1} & =y_{k}-\frac{1+\beta u_{k}+\lambda u_{k}^{2}}{1+(\beta-2) u_{k}+\mu u_{k}^{2}} \frac{f\left(y_{k}\right)}{f^{\prime}\left(x_{k}\right)},
\end{aligned}
$$

where $u_{k}=f\left(y_{k}\right) / f\left(x_{k}\right)$. This family is fourth-order convergent for any value of parameters $\lambda, \beta$, and $\mu$.

Chicharro et $\mathrm{al}^{15}$ made an analysis on a particular case of (1), where $\beta=\mu=0$, resulting in

$$
\begin{aligned}
y_{k} & =x_{k}-\frac{f\left(x_{k}\right)}{f^{\prime}\left(x_{k}\right)}, \\
x_{k+1} & =y_{k}-\frac{1+\lambda u_{k}^{2}}{1-2 u_{k}} \frac{f\left(y_{k}\right)}{f^{\prime}\left(x_{k}\right)},
\end{aligned}
$$

where $u_{k}=f\left(y_{k}\right) / f\left(x_{k}\right)$. Obviously, this family is fourth-order convergent, but it only depends on the parameter $\lambda$. An in-depth analysis of the complex dynamics associated to this family was performed. For $\lambda=1$, it was stated that the corresponding method was stable in terms of dynamics, but for other values of $\lambda$, the corresponding methods had chaotic behavior.

In this paper, we are going to analyze the dynamical behavior of family (1), with $\lambda=1$, selecting the values of parameters $\beta$ and $\mu$ for which the corresponding scheme has good stability properties. This analysis is carried out using complex dynamic tools (Section 2). From the obtained values for $\beta$ and $\mu$, we use accelerating parameters in order to design schemes with memory that increase the order of convergence of the family, without new functional evaluations (Section 3 ). Section 4 covers the real multidimensional dynamics of the resulting family with memory. In this sense, the dynamical planes will show the good stability of the methods with memory. In Section 5, a numerical experience over the best methods of the family is performed and compared with other methods with a similar structure of the literature. Finally, Section 5 gathers the main conclusions of this research.

\section{2 | THE SELECTION OF THE BEST MEMBERS OF THE BIPARAMETRIC FAMILY}

We select the best members of the biparametric Kim's family (1), with $\lambda=1$, in terms of stability, by means of complex dynamics. Therefore, some of its fundamentals are remember below. In Blanchard ${ }^{16}$ and Devaney, ${ }^{17}$ more information about complex dynamics can be found.

\section{1 | Fundamentals on complex dynamics}

Let $R: \hat{\mathbb{C}} \rightarrow \hat{\mathbb{C}}$ be a rational function, where $\widehat{\mathbb{C}}$ represents the Riemann sphere. The orbit of a point $x \in \hat{\mathbb{C}}$ is the set of its images by $R,\left\{x, R(x), R^{2}(x), \ldots, R^{n}(x), \ldots\right\}$. A point $x^{F} \in \hat{\mathbb{C}}$ is called fixed point when $R\left(x^{F}\right)=x^{F}$. Fixed points $x^{F}$ are classified depending on its asymptotic behavior as attracting, repelling, or neutral, depending on whether the value of $\left|R^{\prime}\left(x^{F}\right)\right|$ is less, greater, or equal to 1 , respectively. In addition, $x^{F}$ is called superattracting when $R^{\prime}\left(x^{F}\right)=0$.

For an attractor $x^{*}$, its basin of attraction $\mathcal{A}\left(x^{*}\right)$ is defined as the set of its preimages by $R$ such that

$$
\mathcal{A}\left(x^{*}\right)=\left\{x \in \hat{\mathbb{C}}: R^{n}(x) \rightarrow x^{*}, n \rightarrow \infty\right\} .
$$


A point $x^{C} \in \hat{\mathbb{C}}$ is called critical point when $R^{\prime}\left(x^{C}\right)=0$.

Any iterative method applied on a polynomial $p(x)$ gives us a rational function. In this case, we call strange fixed points, $x^{F}$, those fixed points that are not the roots of the polynomial and free critical points, $x^{C}$, the critical points different of the roots of $p(x)$.

There are several graphical tools that allow to understand the dynamics of an iterative method. The most used is the dynamical plane. Given a set of initial points coverings a region of the plane, the dynamical plane represents the basins of attraction of any attractor: strange fixed points, orbits, etc. This representation can be found in many previous researches. ${ }^{18-20}$

On the other hand, the stability plane is used when we have a biparametric family whose parameters are real or a uniparametric family whose parameter is complex. Given a strange fixed point depending on the involved parameters, its stability plane represents the values of the parameters where the strange fixed point is repelling, attracting, or neutral. Some examples of the use of the stability planes can be found in Cordero et al. ${ }^{21,22}$ Let us remark that, for each strange fixed point, we have its corresponding stability plane.

In order to gather the information of every stability plane, we define the unified plane. This kind of representation has been already used in Chicharro et $\mathrm{al}^{23,24}$ for the illustration of the unified parameters plane, but it can be extended for joining individual lines or planes whose merged information is stronger than the individual one.

Definition 1 (Unified image (line or plane)). Let $P=\left\{(x, y) \in \mathbb{N} \times \mathbb{N}: 1<x<P_{x}, 1<y<P_{y}\right\}$, where $P_{x}$ and $P_{y}$ are the number of pixels of the image $I$ and $C=\{z \in \mathbb{Z}: 0 \leq z \leq 255\}$. Let $I_{R}, I_{G}, I_{B}: P \rightarrow C$ be the intensity of red, green, and blue of the pixels of an image $I$, respectively.

The binary image $\hat{I}: P \rightarrow\{0,1\}$ is defined as

$$
\hat{\mathrm{I}}=\left\{\begin{array}{l}
0, \text { if } I_{R}=I_{G}=I_{B}=0 \\
1, \text { otherwise }
\end{array}\right.
$$

Let $X^{F}=\left\{x_{i}^{F}, i=1,2, \ldots, k,\right\}$ be the set of the $k$ strange fixed points, and let $\hat{F}_{i}$ be their associated binary images, $i=1,2, \ldots, k$. The unified stability image $S: P \rightarrow\{0,1\}$ is defined as

$$
S=\prod_{i=1}^{k} \hat{F}_{i} .
$$

Let $X^{C}=\left\{x_{j}^{C}, j=1,2, \ldots, m\right\}$ be the set of the $m$ free critical points, and let $\hat{C}_{j}$ be their associated binary images. The unified parameters image $\mathcal{P}: P \rightarrow\{0,1\}$ is defined as

$$
\mathcal{P}=\prod_{j=1}^{m} \hat{C}_{j} .
$$

Related with the real dynamics and a uniparametric family, a useful tool is the convergence plane. ${ }^{25}$ This representation gathers in one figure, the dynamical behavior of a set of initial guesses with a set of real values of the parameter, representing the basins of attraction in a similar manner than the dynamical plane does.

\section{2 | Dynamical analysis of the biparametric family}

The biparametric class under study is the original family (1) with $\lambda=1$. Its iterative expression is

$$
\begin{aligned}
y_{k} & =x_{k}-\frac{f\left(x_{k}\right)}{f^{\prime}\left(x_{k}\right)}, \\
x_{k+1} & =y_{k}-\frac{1+\beta u_{k}+u_{k}^{2}}{1+(\beta-2) u_{k}+\mu u_{k}^{2}} \frac{f\left(y_{k}\right)}{f^{\prime}\left(x_{k}\right)}, \quad k=0,1, \ldots
\end{aligned}
$$

where $u_{k}=f\left(y_{k}\right) / f\left(x_{k}\right)$. Throughout this section, we analyze the members of family (3) in order to obtain those with better stability. The complex dynamics of this family will be performed for obtaining the values of parameters $\beta$ and $\mu$ for which wide basins of attraction are getting. 
In the following, we analyze family (3) on a generic quadratic polynomial $p(x)=(x-a)(x-b)$, for $a, b \in \hat{\mathbb{C}}$. The resulting rational function when the iterative family (3) is applied on $p(x)$ is denoted by $O_{\beta, \mu, a, b}(x)$. If we use the Möbius transformation

$$
M(s)=\frac{s-a}{s-b},
$$

the operator $O_{\beta, \mu, a, b}(x)$ is conjugated to operator $O_{\beta, \mu}(x)$,

$$
O_{\beta, \mu}(x)=M \circ O_{\beta, \mu, a, b} \circ M^{-1}(x)=\frac{x^{4}\left(2 \beta+\mu+x^{4}+(\beta+4) x^{3}+x^{2}(4 \beta+\mu+6)+x(5 \beta+2 \mu+4)\right)}{x^{4}(2 \beta+\mu)+x^{3}(5 \beta+2 \mu+4)+x^{2}(4 \beta+\mu+6)+(\beta+4) x+1} .
$$

The previous transformation allows with a suitable scaling to analyze the fixed point operator associated to family (3) on all quadratic polynomials without dependence on parameters $a$ and $b$. The fixed points of operator $O_{\beta, \mu}(x)$ are the values of $x$ such that $O_{\beta, \mu}(x)=x$, that is, $x_{1}=0, x_{2}=\infty$, which correspond to the roots of polynomial $p(x), x_{3}=1$ and the roots of a polynomial of degree 6 .

Proposition 1. The fixed points of $O_{\beta, \mu}(x)$ for $\mu, \beta \in \mathbb{R}$ and their dynamical behavior are as follows:

1. $x_{1}=0$ and $x_{2}=\infty$ are superattracting

2. $x_{3}=1$ is

(a) superattracting, if $\mu=-8-4 \beta$,

(b) attracting, if $\mu \in\left(\frac{-49-5 \beta}{4}, \frac{-79-44 \beta}{12}\right)$,

(c) neutral, if $\mu=-\frac{49}{4}-5 \beta$,

(d) repelling, if $\mu \in\left(-\infty, \frac{-49-5 \beta}{4}\right) \cup\left(\frac{-79-44 \beta}{12},+\infty\right)$,

3. $x_{4-9}(\mu, \beta)$ are the roots of polynomial $s^{6}+s^{5}(\beta+5)+s^{4}(5 \beta+\mu+11)+s^{3}(8 \beta+2 \mu+15)+s^{2}(5 \beta+\mu+11)+s(\beta+5)+1$. Depending on the values of $\mu$ and $\beta$, they behave as attracting, repelling, or saddle points.

Figure 1 gathers the stability planes of $x_{3-9}$ for $\beta, \mu \in \mathbb{R}$. The undesired regions by means of stability are represented in black, since the strange fixed points are attracting. Every plane has been generated using a mesh of $200 \times 200$ points.

Figure 2 represents the unified stability plane of the strange fixed points $x_{3-9}$. Let us remark that the black regions correspond to the real values of $\mu$ and $\beta$ where at least one strange fixed point behaves as an attracting point.

\subsection{Selection of a value for the parameter}

In Figure 2, it is observed that there is a diagonal band where the unified stability plane is in white. It means that there is not any strange fixed point that behaves as an attractor. This band collects the points between the line $\mu=\beta$ and $\mu=\beta+2$, approximately.

Below, some convergence planes are represented in Figure 3 in order to check the information of the unified stability plane and to visualize the wideness of the basins of attraction of the roots for fixed values of $\beta$ and $\mu$. Figure 3A-C represents the convergence planes for $\beta=\mu, \beta=\mu+2$, and $\beta=\mu-5$. Orange and blue colors represent the basins of attraction of the fixed points 0 and $\infty$, respectively. To generate the convergence planes, each point $x_{0}$ of a mesh of 500 points in the interval $[-10,10]$ is taken as initial guess to successively iterate the fixed point function. Moreover, the ordinates axis corresponds to the values of $\beta \in[-10,10]$, so each point in the plane is associated to a pair $\left(x_{0}, \beta\right)$. Then, the point is represented with the corresponding color depending on the basin of attraction where it has converged to. In another case, it is represented in black. The convergence is set when the difference between two consecutive iterates is lower than $10^{-3}$, with a maximum of 50 iterations of the method.

In every case of Figure 3, there are regions where there is no convergence to the roots, as can be seen for $\beta \approx-2.4$ or $\beta \approx-3.2$. It can be motivated by the presence of attracting strange fixed points, as can be deduced from the unified stability plane of Figure 2 for these values of parameters $\beta$ and $\mu$.

Regarding the convergence planes, in Figure 3A,C, there also appear regions of convergence to a point different from the roots. For instance, for $\beta \approx 10$ in Figure 3A, and for $\beta \approx 7$ in Figure 3C. However, in the case of $\mu=\beta+2$, there is no additional region of convergence to a point different from the roots.

Nevertheless, we can try to define curves for which the corresponding convergence planes have greater stability than those considered in Figure 3. In this sense, we must define curves that avoid the black regions present in the unified 


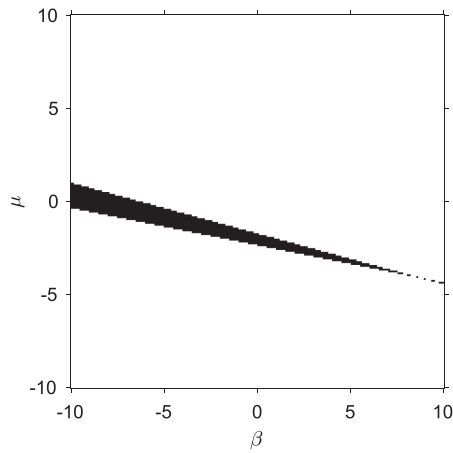

(A) $x_{3}$

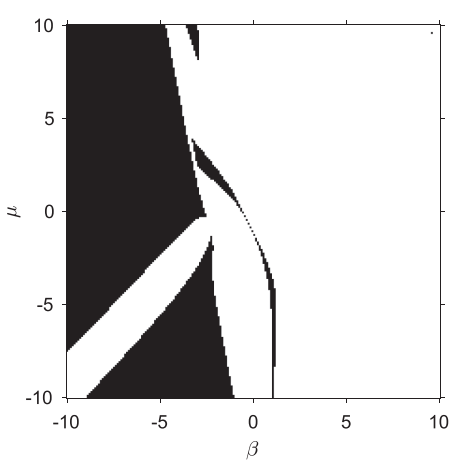

(D) $x_{6}$

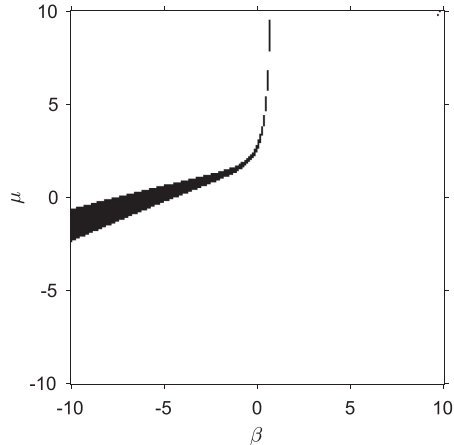

(B) $x_{4}$

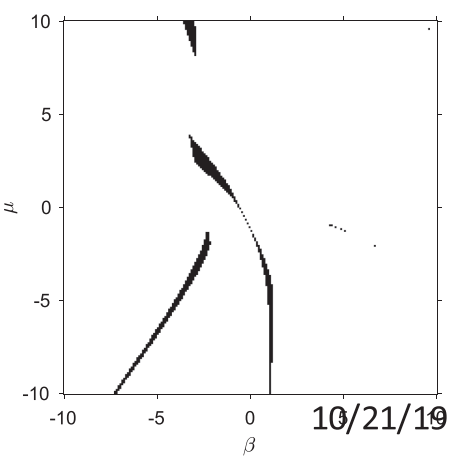

(E) $x_{7}$

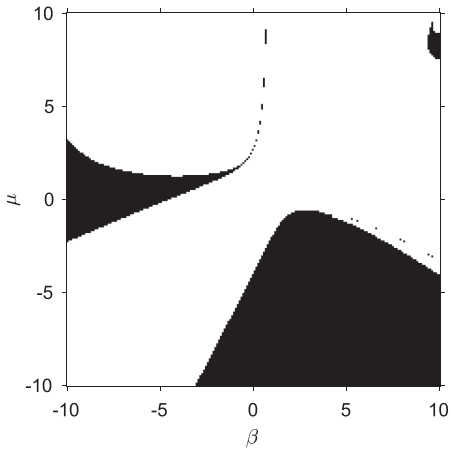

(C) $x_{5}$

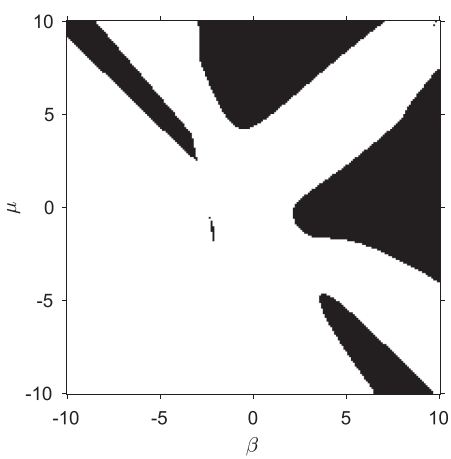

(F) $x_{8-9}$

FIGURE 1 Stability planes of $x_{3-9} \in \mathbb{C}$. The strange fixed points are attracting in the black region

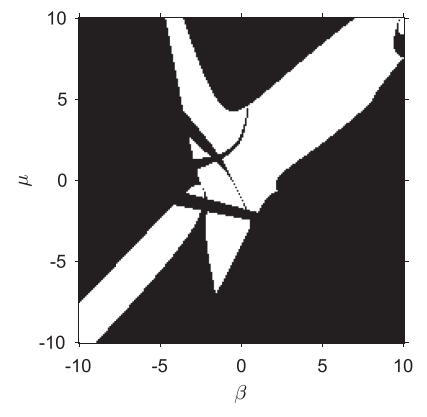

FIGURE 2 Unified stability plane of $x_{3-9} \in \hat{\mathbb{C}}$

stability plane as much as possible for both parameters. Figure 4 represents the parabola $\mu=0.17 \beta^{2}-0.8075 \beta+2.9166$ over the unified stability plane. As we can see, we almost avoid the black regions with this curve. The resulting convergence plane when $\mu=0.17 \beta^{2}-0.8075 \beta+2.9166$ is shown in Figure 5, which reveals that now the family has good stability as all the points converge to a root.

Taking into account the results obtained in Figures 3 and 5, the next section is devoted to develop methods with memory from the uniparametric family corresponding to $\mu=0.17 \beta^{2}-0.8075 \beta+2.9166$.

Then, if we set the value $\mu=P_{2}(\beta)=0.17 \beta^{2}-0.8075 \beta+2.9166$ in (3), we obtain the family of iterative methods

$$
\begin{aligned}
y_{k} & =x_{k}-\frac{f\left(x_{k}\right)}{f^{\prime}\left(x_{k}\right)}, \\
x_{k+1} & =y_{k}-\frac{1+\beta u_{k}+u_{k}^{2}}{1+(\beta-2) u_{k}+P_{2}(\beta) u_{k}^{2}} \frac{f\left(y_{k}\right)}{f^{\prime}\left(x_{k}\right)},
\end{aligned}
$$

where $u_{k}=f\left(y_{k}\right) / f\left(x_{k}\right)$. Its error equation is

$$
e_{k+1}=\left(\left(2 \beta+P_{2}(\beta)\right) c_{2}^{3}-c_{2} c_{3}\right) e_{k}^{4}+\mathcal{O}\left(e_{k}^{5}\right)
$$



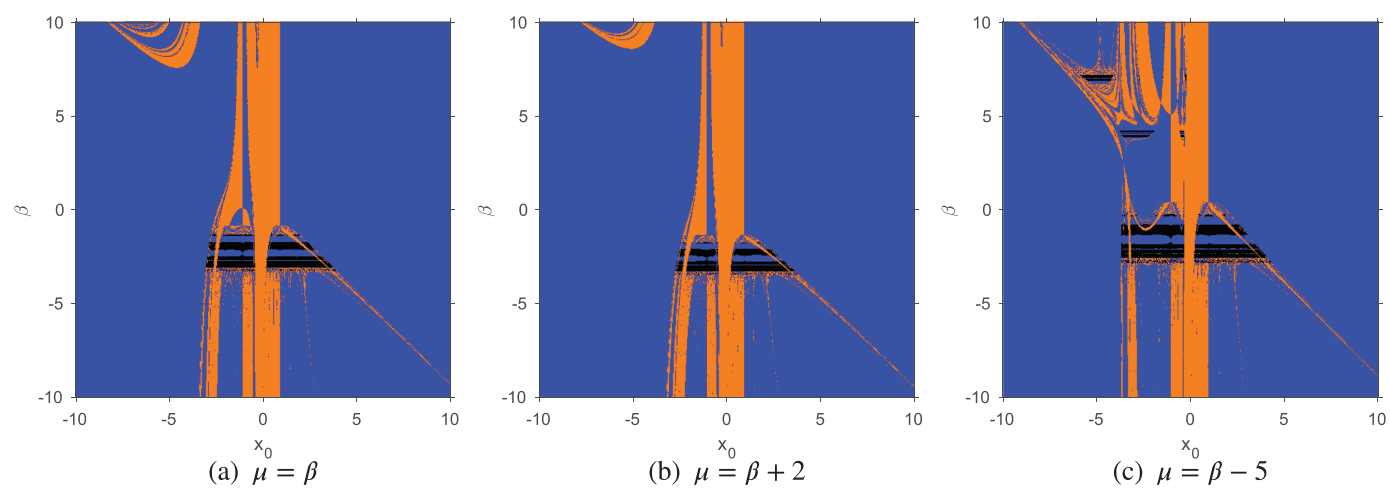

FIGURE 3 Convergence planes for different relations between $\beta$ and $\mu$

FIGURE 4 Unified stability plane of $x_{3-9} \in \mathbb{C}$. In red the curve $\mu=0.17 \beta^{2}-0.8075 \beta+2.9166$

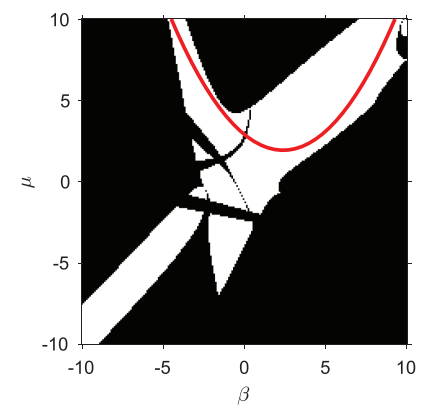

FIGURE 5 Convergence plane for $\mu=0.17 \beta^{2}-0.8075 \beta+2.9166$

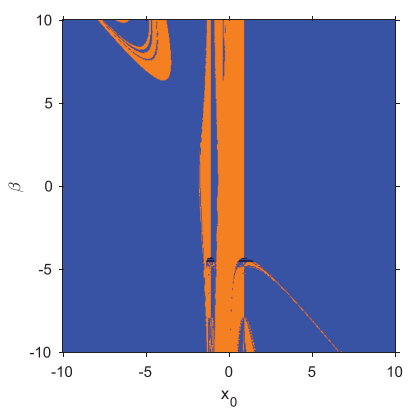

where $c_{j}=\frac{f^{(j)}(\xi)}{j ! f^{\prime}(\xi)}, j \geq 2, e_{k}=x_{k}-\xi, \forall k$ and $\xi$ is the solution of the nonlinear equation $f(x)=0$. As it can be observed in the error equation, family (5) has at least order of convergence four for any value of $\beta$.

\section{3 | ITERATIVE METHODS WITH MEMORY}

In this section, we introduce a new iterative class based on the resulting schemes (5). The proposed family includes memory using self-accelerating parameters and holds a similar expression than the original scheme. It is based on the techniques presented in Chicharro et $\mathrm{al}^{26}$ and Choubey et $\mathrm{al}^{27}$ among others. The following result, which can be found in Ortega and Reinhboldt, ${ }^{28}$ is useful to analyze the order of convergence of a method with memory.

Theorem 1. Let $g$ be the fixed point function of an iterative method with memory that generates a sequence $\left\{x_{k}\right\}$ of approximations to the root $\xi$ of $f(x)=0$, and let this sequence converge to $\xi$. If there exists a nonzero constant $\theta$ and nonnegative numbers $r_{i}, i=0,1, \ldots, m$, such that the following inequality holds

$$
\left|e_{k+1}\right| \leq \theta \prod_{i=0}^{m}\left|e_{k-i}\right|^{r_{i}}
$$


where $e_{k}=x_{k}-\xi, k \geq 0$, denotes the error in each iteration, then the R-order of convergence of the iterative method satisfies the inequality

$$
O_{R}(g, \xi) \geq p^{*}
$$

where $p^{*}$ is the unique positive root of the equation

$$
p^{m+1}-\sum_{i=o}^{m} r_{i} p^{m-i}=0
$$

From family (5) and by using accelerating parameters in each step, we design a new parametric family of iterative schemes whose error equation allows us to introduce memory, which is obtained by approximating the value of accelerating parameters by the use of Padé approximants in the way that we show below. This technique allows to increase the order of convergence without the need of new functional evaluations.

\section{1 | $\operatorname{DFM}(\beta)$ class}

We introduce a self-accelerating parameter $\alpha$ in (5) obtaining a family of iterative schemes who expression is

$$
\begin{aligned}
y_{k} & =x_{k}-\frac{f\left(x_{k}\right)}{f^{\prime}\left(x_{k}\right)+\alpha f\left(x_{k}\right)}, \\
x_{k+1} & =y_{k}-\frac{1+\beta u_{k}+u_{k}^{2}}{1+(\beta-2) u_{k}+P_{2}(\beta) u_{k}^{2}} \frac{f\left(y_{k}\right)}{f^{\prime}\left(x_{k}\right)+2 \alpha f\left(x_{k}\right)}, \quad k=0,1, \ldots,
\end{aligned}
$$

where $u_{k}=f\left(y_{k}\right) / f\left(x_{k}\right)$. Its error equation is

$$
e_{k+1}=\left(\alpha+c_{2}\right)\left(\alpha^{2}\left(2 \beta+P_{2}(\beta)-3\right)+\alpha c_{2}\left(4 \beta+2 P_{2}(\beta)-3\right)+\left(2 \beta+P_{2}(\beta)\right) c_{2}^{2}-c_{3}\right) e_{k}^{4}+\mathcal{O}\left(e_{k}^{5}\right)
$$

If we take $\alpha=-c_{2}=-\frac{f^{\prime \prime}(\xi)}{2 f^{\prime}(\xi)}$, the methods reach, at least, order 5, but the value $\xi$ is unknown. So, we need to obtain some approximations of $f^{\prime}(\xi)$ and $f^{\prime \prime}(\xi)$. We are going to resort to Padé approximants that have been already used in Cordero et $\mathrm{al}^{29}$ and Chicharro et $\mathrm{a}^{30}$ for designing new iterative methods but, as far as we know, they have not been used to estimate accelerating parameters.

The Padé approximant that we propose is

$$
m(t)=\frac{a_{1}+a_{2}\left(t-x_{k}\right)}{1+a_{3}\left(t-x_{k}\right)} .
$$

The coefficients $a_{1}, a_{2}$, and $a_{3}$ are obtained satisfying the conditions

$$
\left\{\begin{array}{l}
m\left(x_{k}\right)=f\left(x_{k}\right), \\
m\left(x_{k-1}\right)=f\left(x_{k-1}\right), \\
m^{\prime}\left(x_{k}\right)=f^{\prime}\left(x_{k}\right)
\end{array}\right.
$$

Solving the system (8), the values of $a_{1}, a_{2}$, and $a_{3}$ are

$$
a_{1}=f\left(x_{k}\right), a_{2}=\frac{f\left(x_{k}\right)^{2}-f\left(x_{k}\right) f\left(x_{k-1}\right)+f^{\prime}\left(x_{k}\right) f\left(x_{k-1}\right)\left(x_{k-1}-x_{k}\right)}{\left(f\left(x_{k}\right)-f\left(x_{k-1}\right)\right)\left(x_{k}-x_{k-1}\right)}, a_{3}=\frac{f\left(x_{k}\right)-f\left(x_{k-1}\right)+f^{\prime}\left(x_{k}\right)\left(x_{k-1}-x_{k}\right)}{\left(f\left(x_{k}\right)-f\left(x_{k-1}\right)\right)\left(x_{k}-x_{k-1}\right)} .
$$

For the approximation of the unknown values, we are using $f^{\prime}(\xi) \approx m^{\prime}\left(x_{k}\right)$ and $f^{\prime \prime}(\xi) \approx m^{\prime \prime}\left(x_{k}\right)$. Therefore, the value of the self-accelerating parameter is

$$
\alpha_{k}=\frac{f\left(x_{k}\right)-f\left(x_{k-1}\right)+f^{\prime}\left(x_{k}\right)\left(x_{k-1}-x_{k}\right)}{\left(f\left(x_{k}\right)-f\left(x_{k-1}\right)\right)\left(x_{k}-x_{k-1}\right)} .
$$

Replacing this value of $\alpha$ in (6), a family of parametric iterative schemes with memory $\operatorname{DFM}(\beta)$ is obtained.

Theorem 2. Let $\xi$ be a simple zero of a sufficiently differentiable function $f: I \subset \mathbb{R} \rightarrow \mathbb{R}$ in an open interval I. If $x_{0}$ is close enough to $\xi$ and $\alpha_{0}$ is given, then the-R order of family $D F M(\beta)$ is at least $2+\sqrt{5} \approx 4.24$ that corresponds to the positive root of polynomial $p^{2}-4 p-1$, and its error equation is 


$$
e_{k+1}=c_{3}\left(c_{3}-c_{2}^{2}\right) e_{k-1} e_{k}^{4}+\mathcal{O}_{6}\left(e_{k-1} e_{k}\right)
$$

where $c_{j}=\frac{f^{(j)}(\xi)}{j ! f^{\prime}(\xi)}, j \geq 2$, and $\mathcal{O}_{6}\left(e_{k-1} e_{k}\right)$ indicates that the sum of the exponents of $e_{k}$ and $e_{k-1}$ in the rejected terms of the development is at least 6 .

Proof. Let us denote by $e_{k}=x_{k}-\xi$, for all $k$, the error in each iteration of family $\operatorname{DFM}(\beta)$. Then, we consider the following Taylor series expansions around $\xi$ :

$$
\begin{aligned}
f\left(x_{k}\right) & =f^{\prime}(\xi)\left[e_{k}+c_{2} e_{k}^{2}+c_{3} e_{k}^{3}+c_{4} e_{k}^{4}+c_{5} e_{k}^{5}\right]+\mathcal{O}\left(e_{k}^{6}\right), \\
f^{\prime}\left(x_{k}\right) & =f^{\prime}(\xi)\left[1+2 c_{2} e_{k}+3 c_{3} e_{k}^{2}+4 c_{4} e_{k}^{3}+5 c_{5} e_{k}^{4}\right]+\mathcal{O}\left(e_{k}^{5}\right), \\
f\left(x_{k-1}\right) & =f^{\prime}(\xi)\left[e_{k-1}+c_{2} e_{k-1}^{2}+c_{3} e_{k-1}^{3}+c_{4} e_{k-1}^{4}+c_{5} e_{k-1}^{5}\right]+\mathcal{O}\left(e_{k-1}^{6}\right),
\end{aligned}
$$

where $c_{j}=\frac{f^{(j)}(\xi)}{j ! f^{\prime}(\xi)}, j \geq 2$.

By using the previous Taylor series expansions, we obtain

$$
\begin{aligned}
\alpha_{k} & =-c_{2}+\left(c_{2}^{2}-c_{3}\right) e_{k-1}+\left(c_{2}^{2}-2 c_{3}\right) e_{k}-2\left(c_{2}^{3}-2 c_{2} c_{3}+c_{4}\right) e_{k-1} e_{k}+\left(-c_{2}^{3}+2 c_{2} c_{3}-c_{4}\right) e_{k-1}^{2}+\left(-c_{2}^{3}+3 c_{2} c_{3}-3 c_{4}\right) e_{k}^{2} \\
& +\mathcal{O}_{3}\left(e_{k-1} e_{k}\right) .
\end{aligned}
$$

Then, we obtain for the first step of $\operatorname{DFM}(\beta)$ class:

$$
\begin{aligned}
y_{k}-\xi & =e_{k}-\frac{f\left(x_{k}\right)}{f^{\prime}\left(x_{k}\right)+\alpha_{k} f\left(x_{k}\right)} \\
& =\left(c_{2}^{2}-c_{3}\right) e_{k-1} e_{k}^{2}+\left(-c_{2}^{3}+2 c_{2} c_{3}-c_{4}\right) e_{k-1}^{2} e_{k}^{2}-2\left(c_{2}^{3}-2 c_{2} c_{3}+c_{4}\right) e_{k-1} e_{k}^{3}+\mathcal{O}_{5}\left(e_{k-1} e_{k}\right) .
\end{aligned}
$$

By expanding $f\left(y_{k}\right)$ around $\xi$, we get

$$
\begin{aligned}
f\left(y_{k}\right) & =f^{\prime}(\xi)\left[y_{k}-\xi+c_{2}\left(y_{k}-\xi\right)^{2}\right]+\mathcal{O}\left(\left(y_{k}-\xi\right)^{3}\right) \\
& =f^{\prime}(\xi)\left[\left(c_{2}^{2}-c_{3}\right) e_{k-1} e_{k}^{2}+\left(-c_{2}^{3}+2 c_{2} c_{3}-c_{4}\right) e_{k-1}^{2} e_{k}^{2}-2\left(c_{2}^{3}-2 c_{2} c_{3}+c_{4}\right) e_{k-1} e_{k}^{3}\right]+\mathcal{O}_{5}\left(e_{k-1} e_{k}\right) .
\end{aligned}
$$

Being $u_{k}=f\left(y_{k}\right) / f\left(x_{k}\right)$, the error equation is

$$
e_{k+1}=c_{3}\left(c_{3}-c_{2}^{2}\right) e_{k-1} e_{k}^{4}+\left(3 c_{2}^{5}-5 c_{2}^{3} c_{3}+c_{2} c_{3}^{2}+c_{3} c_{4}\right) e_{k-1}^{2} e_{k}^{4}+2\left(c_{2}^{2}-2 c_{3}\right)\left(c_{2} c_{3}-c_{4}\right) e_{k-1} e_{k}^{5}+\mathcal{O}_{7}\left(e_{k-1} e_{k}\right)
$$

Finally, using Theorem 1, the order of $\operatorname{DFM}(\beta)$ class is given by the positive root of the equation $p^{2}-4 p-1=0$. Therefore, the order of the family is $p=2+\sqrt{5} \approx 4.24$.

\section{REAL MULTIDIMENSIONAL DYNAMICAL ANALYSIS OF DFM( $(\beta)$ FAMILY}

This section is devoted to perform a dynamical study of family $\operatorname{DFM}(\beta)$ on low degree polynomials. Its rational function on three different quadratic polynomials will be analyzed to obtain the corresponding fixed points and its stability. The basins of attraction for the fixed points will be visualized by representing the dynamical planes.

As it has been studied in Section 3, $\operatorname{DFM}(\beta)$ is a family of iterative methods with memory. In particular, two previous iterates, $x_{k}$ and $x_{k-1}$, are used to obtain the following estimation, $x_{k+1}$, so it has the general form

$$
x_{k+1}=g\left(x_{k-1}, x_{k}\right), \quad k=1,2, \ldots,
$$

being $x_{0}$ and $x_{1}$ the initial estimations and $g$ the operator that defines the family. 
In order to obtain the fixed points of (12), an auxiliary vectorial function from $\mathbb{R}^{2}$ to $\mathbb{R}^{2}$ must be defined. For this purpose, we define the following discrete dynamical system according to the techniques used in previous works ${ }^{31-33}$ :

$$
G(z, x)=(x, g(z, x))
$$

where $z=x_{k-1}$ and $x=x_{k}$. But before starting the dynamical study of family $\operatorname{DFM}(\beta)$, it is necessary to recall some basic concepts about real multidimensional dynamics.

Let us denote by $G$ the rational vectorial fixed point function obtained when a method with memory is applied on a polynomial $p(x)$. The orbit of a point $\vec{x}=(z, x) \in \mathbb{R}^{2}$ is given by the $\operatorname{set}\left\{\vec{x}, G(\vec{x}), G^{2}(\vec{x}) \ldots, G^{n}(\vec{x}), \ldots\right\}$. Fixed points $\vec{x}^{F}$ of (13) are those that satisfy $G\left(\vec{x}^{F}\right)=\vec{x}^{F}$. When a fixed point is not a root of the polynomial, it is called strange fixed point. Moreover, a $m$-periodic point satisfies $G^{m}(\vec{x})=\vec{x}$, being $G^{t}(\vec{x}) \neq \vec{x}$ for $t<m$. The asymptotical behavior of the fixed points is analyzed from the following result. ${ }^{34}$

Theorem 3. Let $G: \mathbb{R}^{n} \rightarrow \mathbb{R}^{n}$ be $\mathcal{C}^{2}$. Let $\lambda_{1}, \lambda_{2}, \ldots, \lambda_{n}$ be the eigenvalues of the Jacobian matrix $G^{\prime}\left(\vec{x}_{m}\right)$, where $\vec{x}_{m}$ is a m-periodic point. Then,

1. If $\left|\lambda_{k}\right|<1$, for all $k, \vec{x}_{m}$ is attracting. In particular, if $\left|\lambda_{k}\right|=0$, for all $k$, it is called superattracting.

2. When $\left|\lambda_{k}\right|>1$, for all $k, \vec{x}_{m}$ is repelling.

3. If one eigenvalue $\lambda_{k_{0}}$ has $\left|\lambda_{k_{0}}\right|>1$, then $\vec{x}_{m}$ is unstable (repelling or saddle).

Moreover, when all the eigenvalues of $G^{\prime}\left(\vec{x}_{m}\right)$ satisfy $\left|\lambda_{k}\right| \neq 1, \vec{x}_{m}$ is called hyperbolic. In addition, it is called hyperbolic saddle point if there exists at least a pair of eigenvalues with $\left|\lambda_{k}\right|>1$ and $\left|\lambda_{l}\right|<1$, for $l, k \in\{1, \ldots, n\}$. Finally, for an attracting fixed point $\vec{x}^{*}$, we define its basin of attraction, $\mathcal{A}\left(\vec{x}^{*}\right)$, as the set of points that satisfy

$$
\mathcal{A}\left(\vec{x}^{*}\right)=\left\{\vec{x} \in \mathbb{R}^{2}: G^{n}(\vec{x}) \rightarrow \vec{x}^{*}, n \rightarrow \infty\right\}
$$

Next, we study the stability of $\operatorname{DFM}(\beta)$ class on the quadratic polynomials $q_{-1}(x)=x^{2}-1, q_{1}(x)=x^{2}+1$ and $q_{0}(x)=x^{2}$. This study allows to generalize the dynamical analysis to any quadratic polynomial since every polynomial of second degree can be reduced to one of these three cases.

\section{1 | Dynamical analysis $\operatorname{DFM}(\beta)$ class}

The rational vectorial fixed point operator obtained when family $\operatorname{DFM}(\beta)$ is applied on $q_{-1}(x)=x^{2}-1$ is

$$
G_{-1, \beta}(z, x)=\left(x, \frac{N_{-1}(z, x, \beta)}{D_{-1}(z, x, \beta)}\right)
$$

where

$$
\begin{aligned}
N_{-1}(z, x, \beta)= & x^{13}\left(z^{2}+1\right)+x^{12} z\left(11 z^{2}+15\right)+x^{11}\left(-\beta+(\beta+44) z^{4}+106 z^{2}+6\right)+x^{10} z\left(-11 \beta+(7 \beta+80) z^{4}\right. \\
& \left.+(4 \beta+398) z^{2}+94\right)+z\left(3 \beta+2 P_{2}(\beta)-z^{6}+z^{4}\left(\beta+2 P_{2}(\beta)+3\right)-4 z^{2}\left(\beta+P_{2}(\beta)\right)\right) \\
& +x^{9}\left(-3 \beta+z^{6}\left(16 \beta+2 P_{2}(\beta)+63\right)+z^{4}\left(35 \beta-4 P_{2}(\beta)+807\right)+z^{2}\left(-48 \beta+2 P_{2}(\beta)+532\right)+28\right) \\
& +x^{2} z\left(37 \beta+12 P_{2}(\beta)+4 z^{6}\left(\beta+P_{2}(\beta)+1\right)+z^{4}\left(-41 \beta+4 P_{2}(\beta)+36\right)+\left(122-20 P_{2}(\beta)\right) z^{2}-6\right) \\
& +x^{8} z\left(-29 \beta+6 P_{2}(\beta)+z^{6}\left(12 \beta+4 P_{2}(\beta)+15\right)+z^{4}\left(97 \beta-2 P_{2}(\beta)+851\right)-2 z^{2}\left(40 \beta+4 P_{2}(\beta)-709\right)\right. \\
& +290)+x\left(5 \beta+4 P_{2}(\beta)+z^{6}\left(4 \beta+6 P_{2}(\beta)-1\right)+z^{4}\left(-21 \beta-8 P_{2}(\beta)+23\right)+2 z^{2}\left(6 \beta-P_{2}(\beta)+3\right)-2\right) \\
& +2 x^{6} z\left(-13 \beta+2 P_{2}(\beta)+z^{6}\left(6 \beta-2 P_{2}(\beta)+50\right)+z^{4}\left(17 \beta+6 P_{2}(\beta)+586\right)-2 z^{2}\left(5 \beta+3 P_{2}(\beta)-452\right)\right. \\
& +176)+x^{3}\left(7 \beta-4 P_{2}(\beta)+z^{6}(-44 \beta+4 P-2(\beta)+52)+z^{4}\left(-55 \beta-12 P_{2}(\beta)+352\right)\right. \\
& \left.+2 z^{2}\left(46 \beta+6 P_{2}(\beta)+83\right)+2\right)+2 x^{7}\left(-3 \beta+2 P_{2}(\beta)+z^{6}\left(46 \beta+6 P_{2}(\beta)+218\right)+z^{4}(-5 \beta\right. \\
& \left.\left.-10 P_{2}(\beta)+926\right)+z^{2}\left(-38 \beta+2 P_{2}(\beta)+548\right)+24\right)+x^{5}\left(-2 \beta-4 P_{2}(\beta)+z^{6}\left(-68 \beta-24 P_{2}(\beta)+346\right)\right. \\
& \left.+2 z^{4}\left(25 \beta+22 P_{2}(\beta)+701\right)+z^{2}\left(20 \beta-16 P_{2}(\beta)+781\right)+45\right)+x^{4} z\left(26 \beta-24 P_{2}(\beta)\right. \\
& \left.-2 z^{6}\left(14 \beta+2 P_{2}(\beta)-5\right)-2 z^{4}\left(49 \beta+8 P_{2}(\beta)-273\right)+z^{2}\left(100 \beta+44 P_{2}(\beta)+723\right)+151\right)
\end{aligned}
$$




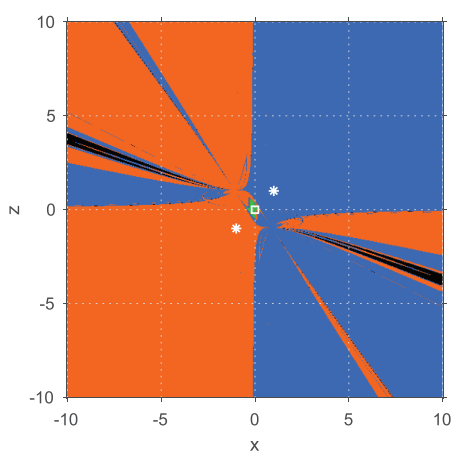

(A) $\beta=6.4$

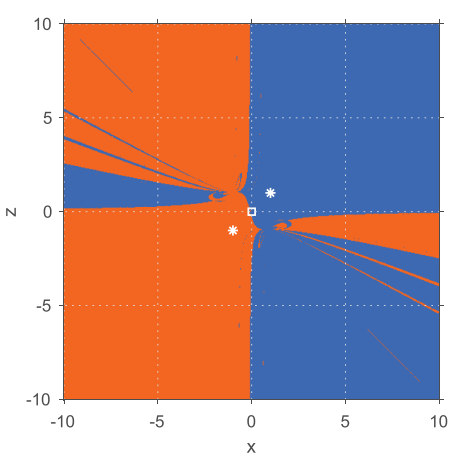

(B) $\beta=4$

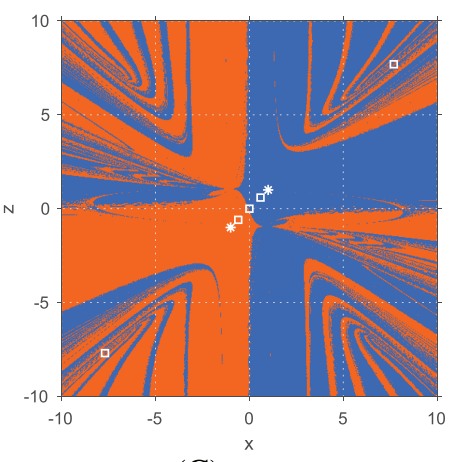

(C) $\beta=67$

FIGURE 6 Dynamical planes of $G_{-1, \beta}$ for different values of $\beta$

and

$$
\begin{aligned}
D_{-1}(z, x, \beta)= & 2(x z+1)\left(x^{2}+2 x z+1\right)^{2}\left(\beta+x^{8}+8 x^{7} z+8 x^{3} z\left(4 z^{2}+3\right)-1+x\left(4 \beta z-4(\beta-2) z^{3}\right)\right. \\
& +x^{6}\left(-\beta+(\beta+22) z^{2}+6\right)+4 x^{5} z\left(-\beta+(\beta+6) z^{2}+8\right)+P_{2}(\beta)+P_{2}(\beta) z^{4} \\
& -z^{2}\left(\beta+2 P_{2}(\beta)-2\right)+x^{4}\left(-\beta+P_{2}(\beta)+z^{4}\left(4 \beta+P_{2}(\beta)+8\right)+z^{2}\left(-3 \beta-2 P_{2}(\beta)+54\right)+8\right) \\
& \left.+x^{2}\left(\beta-2 P_{2}(\beta)-2 z^{4}\left(2 \beta+P_{2}(\beta)-4\right)+z^{2}\left(3 \beta+4 P_{2}(\beta)+18\right)+2\right)\right) .
\end{aligned}
$$

Proposition 2. The fixed points of $G_{-1, \beta}(z, x)$ for $z, x, \beta \in \mathbb{R}$ and their character are as follows:

1. $\vec{x}_{1}=(-1,-1)$ and $\vec{x}_{2}=(1,1)$, which are superattracting,

2. $\vec{x}_{3}=(0,0)$ whose character is

(a) attracting, when $\beta \in[-2.6,-2.58) \cup[6.25,6.65)$,

(b) repelling, if $\beta \in(-2.83,-2.78] \cup(3.97,4.35]$,

(c) a saddle point in other case.

3. $\vec{x}_{4-15}=\left(t_{i}, t_{i}\right)$ where $t_{i}= \pm \sqrt{r_{i}}$, being $r_{1}, r_{2} \ldots, r_{6}$ the roots of the sixth-degree polynomial

$$
\begin{aligned}
P_{6}(x, \beta)= & -29166-6925 \beta+1700 \beta^{2}+(-20000-90000 \beta) x+\left(-735838-181525 \beta-11900 \beta^{2}\right) x^{2} \\
& +\left(-3953328+164600 \beta+13600 \beta^{2}\right) x^{3}+\left(-7407498+319225 \beta+5100 \beta^{2}\right) x^{4} \\
& +\left(-5946672+85400 \beta-13600 \beta^{2}\right) x^{5}+\left(-2387498-290775 \beta+5100 \beta^{2}\right) x^{6} .
\end{aligned}
$$

Among this twelve strange fixed points, only $\vec{x}_{4-9} \in \mathbb{R}$ depending on the value of $\beta$, so we have

(a) $\vec{x}_{4,5} \in \mathbb{R}$ if $\beta \in(6.65,8.74) \cup(8.74,14.61) \cup(14.61,28.4)$, being attracting, repelling, and saddle, in the respective subinterval,

(b) $\vec{x}_{6,7} \in \mathbb{R}$ if $\beta \in(-\infty,-7.3) \cup(-2.58,-2.04) \cup(64.29,+\infty)$. They only are attracting if $\beta \in(-2.57,-2.47)$ and repelling when $\beta \in(-2.47,-2.33)$. If $\beta \in(-\infty,-7.3) \cup(-2.58,-2.57) \cup(-2.33,-2.04)$, they are saddle points,

(c) $\vec{x}_{8,9} \in \mathbb{R}$ if $\beta \in(-\infty,-2.04) \cup(28.42,+\infty)$, being saddle points.

Similar to the convergence planes shown in Section 2, a useful tool to represent the basins of attraction for iterative methods with memory is the dynamical plane.

Figure 6 represents some dynamical planes of $G_{-1, \beta}(z, x)$ for different values of $\beta$. Every dynamical plane has been represented taking a mesh of 500 initial guesses of $x$ and $z$ in the interval $[-10,10] ; 50$ has been the maximum number of iterations involved and $10^{-3}$ the tolerance used as a stopping criterium. We have generated these planes by using the routines described in Chicharro et al. ${ }^{15}$ The basins of attraction of the points $\vec{x}_{1}, \vec{x}_{2}$, and $\vec{x}_{3}$ are mapped with the colors orange, blue, and green, respectively, in every dynamical plane. Depending on the value of $\beta$ considered, the corresponding strange fixed points $\vec{x}_{4-9}$ are also represented in the plane and their basins of attraction in white color. In other cases, the point is plotted in black. White stars represent the fixed points, while the white squares represent the strange fixed points. 
The values of $\beta$ in Figure 6 have been chosen to show different dynamical behaviors of the fixed points and the strange fixed points of $G_{-1, \beta}$, from the results described in Proposition 2. In Figure 6A,B, the operator only has the strange fixed point $\vec{x}_{3}$, which is attracting for $\beta=6.4$ and repelling for $\beta=4$. Then, in addition to the basins of attraction corresponding to the roots of $q_{-1}(x)$, a small basin of attraction of $\vec{x}_{3}$ can be observed in Figure 6A and also two regions without convergence to any fixed point. This does not happen in the other dynamical plane as there is no strange attracting fixed point. In this case, all the points in the plane converge to $\vec{x}_{1}$ or $\vec{x}_{2}$. In Figure $6 \mathrm{C}$, we have represented in white squares the strange fixed points $\vec{x}_{3}$ and $\vec{x}_{6-9}$. Although for $\beta=67$ they are saddle points, we can observe that all the points converge to the superattracting fixed points.

When $\operatorname{DFM}(\beta)$ family is applied on $q_{1}(x)=x^{2}+1$, the resulting rational function is

$$
G_{1, \beta}(z, x)=\left(x, \frac{N_{1}(z, x, \beta)}{D_{1}(z, x, \beta)}\right)
$$

being

$$
\begin{aligned}
N_{1}(z, x, \beta)= & x^{13}\left(z^{2}-1\right)+x^{12} z\left(11 z^{2}-15\right)+x^{11}\left(-\beta+(\beta+44) z^{4}-106 z^{2}+6\right)+x^{10} z\left(-11 \beta+(7 \beta+80) z^{4}\right. \\
& \left.-2(2 \beta+199) z^{2}+94\right)-z\left(3 \beta+2 P_{2}(\beta)+z^{6}+z^{4}\left(\beta+2 P_{2}(\beta)+3\right)+4 z^{2}\left(\beta+P_{2}(\beta)\right)\right) \\
& +x^{9}\left(3 \beta+z^{6}\left(16 \beta+2 P_{2}(\beta)+63\right)+z^{4}\left(-35 \beta+4 P_{2}(\beta)-807\right)+z^{2}\left(-48 \beta+2 P_{2}(\beta)+532\right)-28\right) \\
& +x^{8} z\left(29 \beta-6 P_{2}(\beta)+z^{6}\left(12 \beta+4 P_{2}(\beta)+15\right)+z^{4}\left(-97 \beta+2 P_{2}(\beta)-851\right)-2 z^{2}\left(40 \beta+4 P_{2}(\beta)\right.\right. \\
& -709)-290)-2 x^{6} z\left(13 \beta-2\left(P_{2}(\beta)+88\right)+z^{6}\left(6 \beta-2 P_{2}(\beta)+50\right)-z^{4}\left(17 \beta+6 P_{2}(\beta)+586\right)\right. \\
& \left.-2 z^{2}\left(5 \beta+3 P_{2}(\beta)-452\right)\right)+x\left(-5 \beta-4 P_{2}(\beta)+z^{6}\left(4 \beta+6 P_{2}(\beta)-1\right)+z^{4}\left(21 \beta+8 P_{2}(\beta)-23\right)\right. \\
& \left.+2 z^{2}\left(6 \beta-P_{2}(\beta)+3\right)+2\right)-2 x^{7}\left(3 \beta-2\left(P_{2}(\beta)+12\right)+z^{6}\left(46 \beta+6 P_{2}(\beta)+218\right)\right. \\
& \left.+z^{4}\left(5 \beta+10 P_{2}(\beta)-926\right)+z^{2}\left(-38 \beta+2 P_{2}(\beta)+548\right)\right)-x^{5}\left(-2 \beta-4 P_{2}(\beta)+z^{6}\left(68 \beta+24 P_{2}(\beta)-346\right)\right. \\
& \left.+2 z^{4}\left(25 \beta+22 P_{2}(\beta)+701\right)+z^{2}\left(-20 \beta+16 P_{2}(\beta)-781\right)+45\right)+x^{4} z\left(-26 \beta+24 P_{2}(\beta)-2 z^{6}(14 \beta\right. \\
& \left.\left.+2 P_{2}(\beta)-5\right)+2 z^{4}\left(49 \beta+8 P_{2}(\beta)-273\right)+z^{2}\left(100 \beta+44 P_{2}(\beta)+723\right)-151\right) \\
& +x^{2} z\left(37 \beta+12 P_{2}(\beta)-4 z^{6}\left(\beta+P_{2}(\beta)+1\right)+z^{4}\left(4\left(P_{2}(\beta)+9\right)-41 \beta\right)+2\left(10 P_{2}(\beta)-61\right) z^{2}-6\right) \\
& +x^{3}\left(7 \beta-4 P_{2}(\beta)+z^{6}\left(44 \beta-4\left(p_{2}(\beta)+13\right)\right)+z^{4}\left(-55 \beta-12 P_{2}(\beta)+352\right)-2 z^{2}\left(46 \beta+6 P_{2}(\beta)+83\right)+2\right), \\
D_{1}(z, x, \beta)= & 2(x z-1)\left(x^{2}+2 x z-1\right)^{2}\left(x^{8}+8 x^{7} z-8 x^{3} z\left(4 z^{2}-3\right)-1+\beta-4 x z\left(\beta+(\beta-2) z^{2}\right)\right. \\
& +x^{6}\left(\beta+(\beta+22) z^{2}-6\right)+4 x^{5} z\left(\beta+(\beta+6) z^{2}-8\right)+P_{2}(\beta)+P_{2}(\beta) z^{4}+z^{2}\left(\beta+2 P_{2}(\beta)-2\right) \\
& +x^{4}\left(-\beta+P_{2}(\beta)+z^{4}\left(4 \beta+P_{2}(\beta)+8\right)+z^{2}\left(3 \beta+2 P_{2}(\beta)-54\right)+8\right) \\
& \left.+x^{2}\left(-\beta+2 P_{2}(\beta)+2 z^{4}\left(2 \beta+P_{2}(\beta)-4\right)+z^{2}\left(3 \beta+4 P_{2}(\beta)+18\right)-2\right)\right) .
\end{aligned}
$$

Let us note that now the polynomial under study has no real roots, so all the fixed points of its corresponding operator will be strange fixed points.

Proposition 3. The strange fixed points of $G_{1, \beta}(z, x)$ and their stability are as follows:

1. $\vec{y}_{1}=(0,0)$ whose character is

(a) attracting, if $\beta \in[-2.6,-2.58) \cup[6.25,6.65)$,

(b) repelling, if $\beta \in(-2.83,-2.78) \cup(3.97,4.35]$,

(c) saddle in other case.

2. $\vec{y}_{2-13}=\left(l_{i}, l_{i}\right)$, for $l_{i}= \pm \sqrt{s_{i}}$, where $s_{1}, \ldots, s_{6}$ are the roots of the following polynomial:

$$
\begin{aligned}
Q_{6}(x, \beta)= & -29166-6925 \beta+1700 \beta^{2}+(20000+90000 \beta) x-+\left(-735838-181525 \beta-11900 \beta^{2}\right) x^{2} \\
& +\left(3953328-164600 \beta-13600 \beta^{2}\right) x^{3}+\left(-7407498+319225 \beta+5100 \beta^{2}\right) x^{4} \\
& +\left(5946672-85400 \beta+13600 \beta^{2}\right) x^{5}+\left(-2387498-290775 \beta+5100 \beta^{2}\right) x^{6} .
\end{aligned}
$$

However, $\vec{y}_{10-13} \in \mathbb{C}$ for all $\beta$. Regarding the stability of the real strange fixed points, we obtain

(a) $\vec{y}_{2,3} \in \mathbb{R}$ if $\beta \in(-2.04,-1.32) \cup(4.14,6.65)$, where they are saddle points,

(b) $y \rightarrow 4,5 \in R$ if $\beta \in(-7.28,-2.58) \cup(-2.04,-2) \cup(-2,-1.32) \cup(4.14,64.29)$, being saddle in all the subintervals except for $\beta \in(-2,-1.32)$, where they are repelling, 


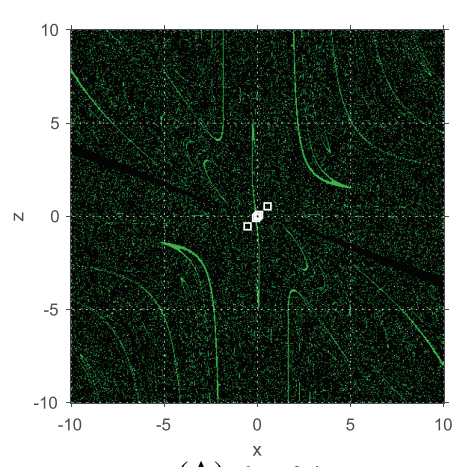

(A) $\beta=6.4$

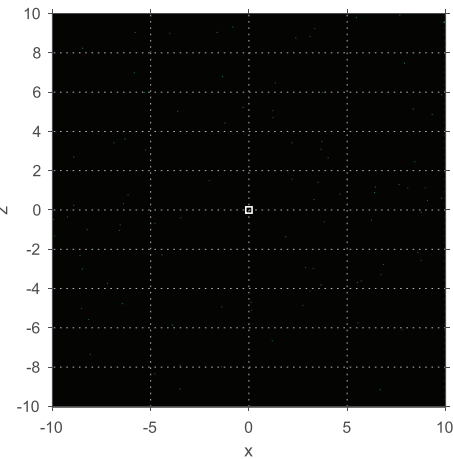

(B) $\beta=4$

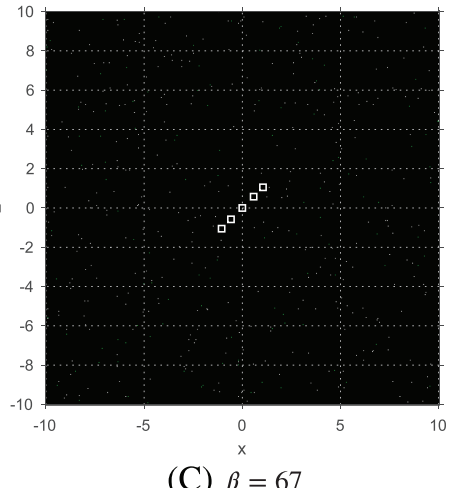

(C) $\beta=67$

FIGURE 7 Dynamical planes of $G_{1, \beta}$ for different values of $\beta$

FIGURE 8 Dynamical planes of $G_{1, \beta}$ for $\beta=6.4$ and $[x, z] \in[-1,1] \times[-1,1]$

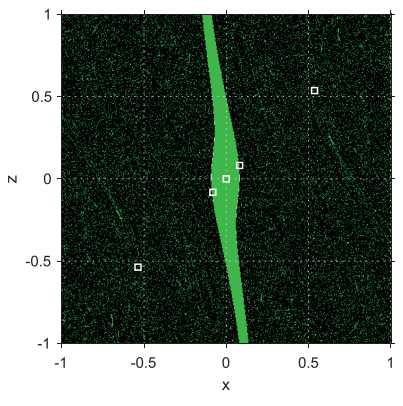

(c) $\vec{y}_{6,7} \in \mathbb{R}$ when $\beta \in(-\infty,-6.24) \cup(-6.24,-2.04) \cup(28.4,64.29) \cup(64.29,+\infty)$, being repelling for $\beta \in$ $(28.4,64.29)$ and saddle in other cases,

(d) $\vec{y}_{8,9} \in \mathbb{R}$ if $\beta \in(-\infty,-2.04) \cup(28.4,64.29) \cup(64.29,+\infty)$ that are saddle points when $\beta \in(-\infty,-2.04) \cup$ $(28.4,64.29)$ and repelling for $\beta \in(64.29,+\infty)$.

Dynamical planes of $G_{1, \beta}$ are showed in Figure 7 for different values of $\beta$. Following the same color notation than Figure 6 , the basin of attraction of $\vec{y}_{1}$ is represented in green, while for $\vec{y}_{2-9}$, their basins of attraction are represented in white. As all the fixed points are strange fixed points, they are plotted with white squares for the values of $\beta$ where they are defined according to Proposition 3.

The values of $\beta$ chosen to generate the planes in Figure 7 correspond to regions where the asymptotic behavior of $\vec{y}_{1}$ is different, that is, attracting, repelling, or saddle for Figure 7A,B,C, respectively. In addition to $\vec{y}_{1}$, for $\beta=6.4$, the operator has the strange fixed points $\vec{y}_{2-5}$, being saddle, while for $\beta=67$, it has the saddle points $\vec{y}_{6,7}$ and the repelling points $\vec{y}_{8,9}$, all of them represented with white squares. This fact is observed in Figure $7 \mathrm{C}$, where the presence of saddle points generates that there are some points distributed on the plane that converge to them, since they are plotted in white or green. Instead, the dynamical plane of Figure 7B is black, so there is no convergence to any strange fixed point.

Figure 8 shows a zoom of the dynamical plane of Figure 7B. There we can see a larger basin of attraction of $\vec{y}_{1}$ than in the other cases because this strange point is attracting for $\beta=6.4$. Moreover, additional white points appear in Figure 8 that correspond to basins of attraction associated to the saddle points $\vec{y}_{2-5}$.

The observed results in Figures 7 and 8 agree with the expected behavior from Proposition 3.

Finally, the fixed point operator corresponding to polynomial $q_{0}(x)=x^{2}$ is

$$
G_{0, \beta}(z, x)=\left(x, \frac{N_{0}(z, x, \beta)}{D_{0}(z, x, \beta)},\right)
$$

where

$$
\begin{aligned}
N_{0}(z, x, \beta)= & x z\left(x^{5}+11 x^{4} z+\beta x^{3} z^{2}+44 x^{3} z^{2}+7 \beta x^{2} z^{3}+80 x^{2} z^{3}+16 \beta x z^{4}\right. \\
& \left.+2 P_{2}(\beta) x z^{4}+63 x z^{4}+12 \beta z^{5}+4 P_{2}(\beta) z^{5}+15 z^{5}\right),
\end{aligned}
$$

and

$$
D_{0}(z, x, \beta)=2(x+2 z)^{2}\left(x^{4}+8 x^{3} z+\beta x^{2} z^{2}+22 x^{2} z^{2}+4 \beta x z^{3}+24 x z^{3}+4 \beta z^{4}+P_{2}(\beta) z^{4}+8 z^{4}\right) .
$$




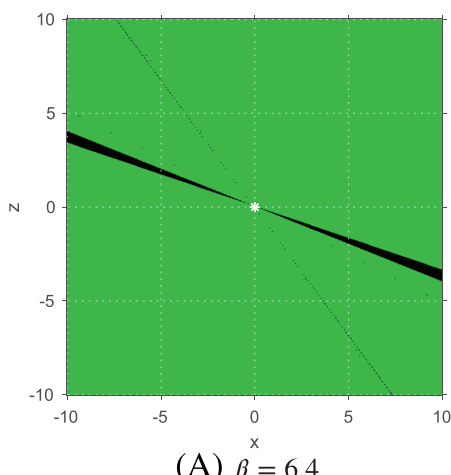

(A) $\beta^{\mathrm{x}}=6.4$

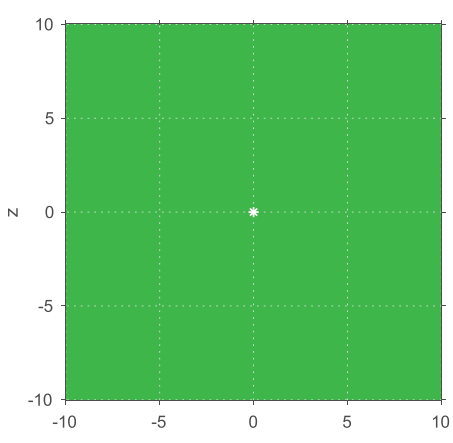

(B) $\stackrel{x}{\beta}=4$

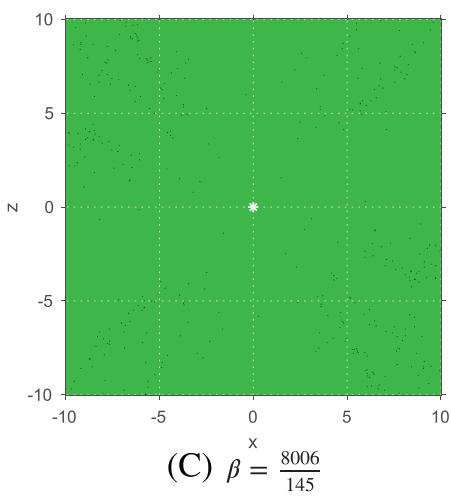

FIGURE 9 Dynamical planes of $G_{0, \beta}$ for different values of $\beta$

The only real fixed point of operator $G_{0, \beta}(z, x)$ is the root of polynomial $q_{0}(x)$. Its stability is analyzed in the following result.

Proposition 4. The only fixed point of $G_{0, \beta}(z, x)$ is $\vec{w}_{1}=(0,0)$ for $\beta \in \mathbb{R} \backslash\left\{-\frac{5281}{752}, \frac{8006}{145}\right\}$. It is attracting in all the points of its domain.

Figure 9 shows some dynamical planes of $G_{0, \beta}$. The only fixed point of the operator, $\vec{w}_{1}$, is represented with a white star and its basin of attraction in green.

\section{5 | NUMERICAL EXPERIENCE}

This section is devoted to show the features of several elements of the family $\operatorname{DFM}(\beta)$. We have chosen four values of parameter $\beta$, which is four members of the family, from the results obtained in the previous section. From Proposition 2 , we choose $\beta=6.4$ and $\beta=8$ as values of the parameter whose corresponding iterative schemes have bad dynamical behavior. In the other sense, we select $\beta=4$ and $\beta=67$ that correspond to stable members of the family. The nonlinear test functions that we use and the obtained zeros are the following:

- $f_{1}(x)=\arctan x, \xi=0$,

- $f_{2}(x)=\cos x-x e^{x}+x^{2}, \xi \approx 0.639154$,

- $f_{3}(x)=\frac{x}{1-x}-5 \ln (0.4(1-x) /(0.4-0.5 x))+4.45977, \xi_{1} \approx 0.757397, \xi_{2} \approx 1.098984$,

- $f_{4}(x)=(x-1)^{3}-1, \xi=2$,

- $f_{5}(x)=e^{x} \sin (5 x)-2, \xi \approx 1.363973$.

\begin{tabular}{llllll}
$\boldsymbol{x}_{\mathbf{0}}$ & $\boldsymbol{\beta}$ & Iter & $\left|\boldsymbol{x}_{\boldsymbol{k}+\mathbf{1}}-\boldsymbol{x}_{\boldsymbol{k}}\right|$ & $\boldsymbol{f}\left(\boldsymbol{x}_{\boldsymbol{k}+\mathbf{1}}\right) \mid$ & ACOC \\
1 & 6.4 & n.c. & - & - & - \\
1 & 4 & 7 & $2.54 \mathrm{e}-64$ & $6.77 \mathrm{e}-271$ & 4.227 \\
1 & 67 & 6 & $1.33 \mathrm{e}-94$ & $3.75 \mathrm{e}-399$ & 4.255 \\
1 & 8 & n.c. & - & - & - \\
0.5 & 6.4 & 4 & $1.65 \mathrm{e}-99$ & $6.01 \mathrm{e}-420$ & 4.235 \\
0.5 & 4 & 4 & $1.28 \mathrm{e}-134$ & $1.137 \mathrm{e}-568$ & 4.235 \\
0.5 & 67 & 5 & $2.77 \mathrm{e}-156$ & $1.86 \mathrm{e}-660$ & 4.232 \\
0.5 & 8 & 4 & $2.13 \mathrm{e}-68$ & $3.69 \mathrm{e}-288$ & 4.243 \\
\hline
\end{tabular}

TABLE 1 Numerical results for several iterative methods with memory on $f_{1}(x)$

$\begin{array}{llllll}\boldsymbol{x}_{\mathbf{0}} & \beta & \text { Iter } & \left|\boldsymbol{x}_{\boldsymbol{k}+\mathbf{1}}-\boldsymbol{x}_{\boldsymbol{k}}\right| & \left|\boldsymbol{f}\left(\boldsymbol{x}_{\boldsymbol{k}+\mathbf{1}}\right)\right| & \text { ACOC } \\ 0 & 6.4 & 8 & 1.52 \mathrm{e}-172 & 4.56 \mathrm{e}-729 & 4.238 \\ 0 & 4 & 6 & 4.24 \mathrm{e}-185 & 8.18 \mathrm{e}-781 & 4.237 \\ 0 & 67 & 5 & 3.72 \mathrm{e}-90 & 4.24 \mathrm{e}-380 & 4.190 \\ 0 & 8 & 10 & 9.15 \mathrm{e}-61 & 1.37 \mathrm{e}-255 & 4.180 \\ 10 & 6.4 & 9 & 3.06 \mathrm{e}-97 & 4.43 \mathrm{e}-410 & 4.233 \\ 10 & 4 & 9 & 3.63 \mathrm{e}-132 & 5.14 \mathrm{e}-558 & 4.242 \\ 10 & 67 & 11 & 2.14 \mathrm{e}-184 & 2.81 \mathrm{e}-779 & 4.232 \\ 10 & 8 & 9 & 1.20 \mathrm{e}-82 & 2.94 \mathrm{e}-348 & 4.226\end{array}$

TABLE 2 Numerical results for several iterative methods with memory on $f_{2}(x)$ 
TABLE 3 Numerical results for several iterative methods with memory on $f_{3}(x)$

\begin{tabular}{llllll}
$\boldsymbol{x}_{\mathbf{0}}$ & $\beta$ & Iter & $\left|\boldsymbol{x}_{\boldsymbol{k}+\mathbf{1}}-\boldsymbol{x}_{\boldsymbol{k}}\right|$ & $\left|\boldsymbol{f}\left(\boldsymbol{x}_{\boldsymbol{k}+\mathbf{1}}\right)\right|$ & ACOC \\
0 & 6.4 & 9 & $3.72 \mathrm{e}-107$ & $8.92 \mathrm{e}-447$ & 4.248 \\
0 & 4 & 9 & $9.17 \mathrm{e}-178$ & $6.95 \mathrm{e}-746$ & 4.239 \\
0 & 67 & 8 & $8.98 \mathrm{e}-67$ & $9.90 \mathrm{e}-276$ & 4.223 \\
0 & 8 & 8 & $2.12 \mathrm{e}-49$ & $2.99 \mathrm{e}-202$ & 3.950 \\
2 & 6.4 & 9 & $2.22 \mathrm{e}-131$ & $7.84 \mathrm{e}-550$ & 4.232 \\
2 & 4 & 8 & $3.36 \mathrm{e}-61$ & $1.55 \mathrm{e}-252$ & 4.233 \\
2 & 67 & 9 & $2.27 \mathrm{e}-187$ & $5.48 \mathrm{e}-787$ & 4.245 \\
2 & 8 & 9 & $1.85 \mathrm{e}-165$ & $3.44 \mathrm{e}-694$ & 4.232 \\
\hline
\end{tabular}

TABLE 4 Numerical results for several iterative methods with memory on $f_{4}(x)$

\begin{tabular}{llllll}
$\boldsymbol{x}_{\mathbf{0}}$ & $\boldsymbol{\beta}$ & Iter & $\left|\boldsymbol{x}_{\boldsymbol{k}+\mathbf{1}}-\boldsymbol{x}_{\boldsymbol{k}}\right|$ & $\left|\boldsymbol{f}\left(\boldsymbol{x}_{\boldsymbol{k}+\mathbf{1}}\right)\right|$ & ACOC \\
1.5 & 6.4 & 46 & $7.17 \mathrm{e}-97$ & $4.37 \mathrm{e}-409$ & 4.189 \\
1.5 & 4 & 5 & $3.10 \mathrm{e}-174$ & $8.97 \mathrm{e}-736$ & 4.234 \\
1.5 & 67 & 8 & $5.36 \mathrm{e}-153$ & $7.88 \mathrm{e}-646$ & 4.219 \\
1.5 & 8 & n.c. & - & - & - \\
0 & 6.4 & 77 & $4.35 \mathrm{e}-96$ & $8.77 \mathrm{e}-405$ & 4.178 \\
0 & 4 & 7 & $1.81 \mathrm{e}-198$ & $2.00 \mathrm{e}-838$ & 4.238 \\
0 & 67 & 8 & $2.37 \mathrm{e}-116$ & $1.01 \mathrm{e}-490$ & 4.194 \\
0 & 8 & n.c. & - & - & - \\
\hline
\end{tabular}

TABLE 5 Numerical results for several iterative methods with memory on $f_{5}(x)$

\begin{tabular}{llllll}
$\boldsymbol{x}_{\mathbf{0}}$ & $\boldsymbol{\beta}$ & Iter & $\left|\boldsymbol{x}_{\boldsymbol{k}+\mathbf{1}}-\boldsymbol{x}_{\boldsymbol{k}}\right|$ & $\left|\boldsymbol{f}\left(\boldsymbol{x}_{\boldsymbol{k}+\mathbf{1}}\right)\right|$ & ACOC \\
1.5 & 6.4 & 8 & $8.77 \mathrm{e}-157$ & $1.94 \mathrm{e}-659$ & 4.237 \\
1.5 & 4 & 4 & $2.13 \mathrm{e}-50$ & $8.88 \mathrm{e}-209$ & 4.260 \\
1.5 & 67 & 5 & $1.25 \mathrm{e}-109$ & $1.08 \mathrm{e}-459$ & 4.238 \\
1.5 & 8 & 6 & $9.89 \mathrm{e}-91$ & $5.76 \mathrm{e}-379$ & 4.198 \\
0.5 & 6.4 & n.c. & - & - & - \\
0.5 & 4 & 9 & $4.77 \mathrm{e}-74$ & $5.90 \mathrm{e}-309$ & 4.255 \\
0.5 & 67 & 30 & $5.25 \mathrm{e}-194$ & $4.00 \mathrm{e}-817$ & 4.232 \\
0.5 & 8 & n.c. & - & - & - \\
\hline
\end{tabular}

In Tables 1 to 5, we present the numerical results obtained for the different test functions. Numerical computations have been carried out in Matlab R2014b and a processor Intel(R) Xeon(R) CPU E5-2420 v2 at $2.20 \mathrm{GHz}$, with variable precision arithmetics and 2000 digits of mantissa. The stopping criterium used is either $\left|x_{k+1}-x_{k}\right|<10^{-200}$ or $\left|f\left(x_{k+1}\right)\right|<10^{-200}$, and both values are displayed in each table, as well as the number of iterations and the approximated computational order of convergence ACOC, ${ }^{35}$ obtained by

$$
A C O C=\frac{\ln \left(\left|x_{k+1}-x_{k}\right| /\left|x_{k}-x_{k-1}\right|\right)}{\ln \left(\left|x_{k}-x_{k-1}\right| /\left|x_{k-1}-x_{k-2}\right|\right)} .
$$

As we need two initial approximations for starting the iterative process, we use $x_{0}$ as initial estimation and with $\alpha_{0}=0.01$ we construct the next iterate $x_{1}$. We denote by n.c. when the method is not convergent for a particular $x_{0}$ and after that 200 iterations.

In Table 1, we show the numerical results for $f_{1}(x)=\arctan x$ and two initial estimations. For $x_{0}=1$, the methods corresponding to $\beta=6.4$ and $\beta=8$ are not convergent, whereas we obtain good results for $\beta=4$ and $\beta=67$, confirming the results proved in Section 4. For the other initial approximation $x_{0}=0.5$, although all methods are convergent, we observe that the error bounds represented by $\left|x_{k+1}-x_{k}\right|$ and $\left|f\left(x_{k+1}\right)\right|$ are better for some parameter values than others. We can find a similar situation in Table 2 for function $f_{2}(x)=\cos x-x e^{x}+x^{2}$ and initial guesses $x_{0}=0$ and $x_{0}=10$.

Table 3 shows the values obtained for function $f_{3}(x)=\frac{x}{1-x}-5 \ln (0.4(1-x) /(0.4-0.5 x))+4.45977$. If we use $x_{0}=0$, then the methods converge to $\xi_{1}$, while with $x_{0}=2$, the convergence is to $\xi_{2}$. In both cases, we observe better error bounds for $\beta=4$ and $\beta=67$ than for $\beta=6.4$ and $\beta=8$.

In Table 4, we highlight the high number of iterations needed for $\beta=6.4$ and the nonconvergence for $\beta=8$, compared with the good results for the other values of the parameter. Finally, Table 5 describes the numerical results for function $f_{5}(x)=e^{x} \sin (5 x)-2$. For $x_{0}=0.5$, the methods corresponding to $\beta=6.4$ and $\beta=8$ are not convergent. However, for $x_{0}=1.5$, all the methods converge and the best results for the error bounds correspond to $\beta=6.4$, perhaps because in this case we use more iterations. 


\section{6 | CONCLUSIONS}

By using complex dynamical tools, we select a parametric subfamily of the Kim class of iterative methods for solving nonlinear equations, with good stability properties. By introducing accelerating parameters and approximating them by Padé approximants, a family of iterative schemes with memory is presented. The real multidimensional dynamics allows us to show that for wide regions of variation the parameter, the corresponding members of this family have good stability properties whereas for a few values of beta, the iterative schemes with memory have chaotic behavior. These results are confirmed with several numerical examples solved with different members of the family with values for the parameter chosen from the results of the dynamic study.

\section{ACKNOWLEDGEMENTS}

This work does not have any conflicts of interest. This research was partially supported by PGC2018-095896-B-C22 (MCIU/AEI/FEDER, UE) and Generalitat Valenciana PROMETEO/2016/089.

\section{ORCID}

Francisco Israel Chicharro López (iD) https://orcid.org/0000-0001-9116-2870

Alicia Cordero (D) https://orcid.org/0000-0002-7462-9173

Juan Ramon Torregrosa (iD https://orcid.org/0000-0002-9893-0761

\section{REFERENCES}

1. Amat S, Busquier S. On the Design of Optimal Iterative Methods for Solving Nonlinear Equations. Switzerland: Springer; 2016.

2. Petković MS, Neta B, Petković LD, Džunić J. Multipoint Methods for Solving Nonlinear Equations. Amsterdam, The Netherlands: Academic Press; 2013.

3. Kung HT, Traub JF. Optimal order of one-point and multipoint iteration. J Assoc Comput Math. 1974;21:643-651.

4. Steffensen JF. Remarks on iteration. Skand Aktuar Tidsr. 1933;16:64-72.

5. Traub JF. Iterative Methods for the Solution of Equations. Englewood Cliffs, New Jersey: Prentice-Hall; 1964.

6. Džunić J, Petković MS, Petković LD. Three-point methods with and without memory for solving nonlinear equations. Appl Math Comput. 2012;218:4917-4927.

7. Džunić J, Petković MS. On generalized biparametric multipoint root finding methods with memory. J Comput Appl Math. 2014;255:362-375.

8. Džzunić J, Petković LD, Petković MS. On an application of Herzberger's matrix method to multipoint families of root-solvers. Filomat. 2018;32:11:3815-3829.

9. Lotfi T, Soleymani F, Assari P. On the construction of some tri-parametric iterative methods wit memory. Numer Algor. 2015;70:835-845.

10. Sharma JR, Gupta P. On some highly efficient derivative free methods with and without memory for solving nonlinear equations. Int $J$ Comput Methods. 2015;12:1-28.

11. Zafar F, Yasmin N, Kutbi MA, Zeshan M. Construction of tri-parametric derivative free fourth order with and without memory iterative method. J Nonlinear Sci Appl. 2016;9:1410-1423.

12. Zafar F, Cordero A, Torregrosa JR, Rafi A. A class of four parametric with- and without-memory root finding methods. Comp and Math Methods. 2019;1(3):1-14. e1024.

13. Torkashvand V, Lotfi T, Araghi MAF. A new family of adaptive methods with memory for solving nonlinear equations. Math Sci. 2019;13:1-20.

14. Kim YI. A triparametric family of three-step optimal eighth-order methods for solving nonlinear equations. Int J Comput Math. 2012;89:1051-1059.

15. Chicharro FI, Cordero A, Torregrosa JR. Drawing dynamical and parameters planes of iterative families and methods. Sci World J. 2013;780153:1-11.

16. Blanchard P. Complex analytic dynamics on the Riemann sphere. Bull AMS. 1984;11:85-141.

17. Devaney RL. An Introduction to Chaotic Dynamical Systems. Colorado, USA: Addison-Wesley; 1989.

18. Sharma JR, Arora H. A new family of optimal eighth order methods with dynamics for nonlinear equations. Appl Math Comput. 2016;273:924-933.

19. Lee SD, Kim YI, Neta B. An optimal family of eighth-order simple-root finders with weight functions dependent on function-to-function ratios and their dynamics underlying extraneous fixed points. J Comput Appl Math. 2017;317:31-54.

20. Geum YH, Kim YI, Neta B. Developing an optimal class of generic sixteenth-order simple-root finders and investigating their dynamics. Math. 2019;7:8.https://doi.org/10.3390/math7010008

21. Cordero A, Gutiérrez JM, Magreñán ÁA, Torregrosa JR. Stability analysis of a parametric family of iterative methods for solving nonlinear models. Appl Math Comput. 2016;285:26-40. 
22. Cordero A, Guasp L, Torregrosa JR. Choosing the most stable members of Kou's family of iterative methods. $J$ Comput Appl Math. 2018;330:769-769.

23. Chicharro FI, Cordero A, Torregrosa JR, Vassileva MP. King-type derivative-free iterative families: real and memory dynamics. Complex. 2017;2713145:1-15.

24. Chicharro FI, Cordero A, Torregrosa JR. High-order extension of an efficient iterative method for solving nonlinear problems. AIP Proc. 2018;1978:330002.

25. Magreñán ÁA. A new tool to study real dynamics: the convergence plane. Appl Math Comput. 2014;248:215-224.

26. Chicharro FI, Cordero A, Garrido N, Torregrosa JR. Stability and applicability of iterative methods with memory. J Math Chem. 2019;57:1282-1300.

27. Choubey N, Cordero A, Jaiswal JP, Torregrosa JR. Dynamical techniques for analyzing iterative schemes with memory. Complexity. 2018;1231341:1-13.

28. Ortega JM, Reinhboldt WC. Iterative Solution of Nonlinear Equations in Several Variables. New York, USA: Academic Press; 1970.

29. Cordero A, Hueso JL, Martínez E, Torregrosa JR. A new technique to obtain derivative-free optimal iterative methods for solving nonlinear equations. J Comput Appl Math. 2013;252:95-102.

30. Chicharro FI, Cordero A, Torregrosa JR. Dynamics and fractal dimension of Steffensen-type methods. Algorithms. 2015;8:271-279.

31. Campos B, Cordero A, Torregrosa JR, Vindel P. A multidimensional dynamical approach to iterative methods with memory. Appl Math Comput. 2015;318:701-715.

32. Campos B, Cordero A, Torregrosa JR, Vindel P. Stability of King's family of iterative methods with memory. J Comput Appl Math. 2017;318:504-514.

33. Chicharro FI, Cordero A, Garrido N, Torregrosa JR. Anomalies in the convergence of Traub-type methods with memory. Comp and Math Methods. 2019;e1060:1-13.

34. Robinson RC. An Introduction to Dynamical Systems: Continuous and Discrete. Rhode Island, USA: American Mathematical Society; 2012.

35. Cordero A, Torregrosa JR. Variants of Newton's method using fifth order quadrature formulas. Appl Math Comput. 2007;190:686-698. 